\title{
Parameter study of star-disc encounters
}

\begin{abstract}
S. Pfalzner, P. Vogel, J. Scharwächter, and C. Olczak
I. Physikalisches Institut, University of Cologne, Germany

Received 2 December 2004 / Accepted 5 April 2005

Abstract. Interactions between disc-surrounded stars might play a vital role in the formation of planetary systems. Here a first parameter study of the effects of encounters on low-mass discs is presented. The dependence of the mass and angular momentum transport on the periastron distance, the relative mass of the encountering stars and eccentricity of the encounter is investigated in detail. This is done for prograde and retrograde coplanar encounters as well as non-coplanar encounters. For distant coplanar encounters our simulation results agree with the analytical approximation of the angular momentum loss by Ostriker (1994, ApJ, 424, 292). However, for close or high-mass encounters, significant differences to this approximation are found. This is especially so in the case of retrograde encounters, where the analytical result predicts no angular momentum loss regardless of the periastron distance whereas the simulations find up to $\sim 20 \%$ loss for close encounters. For the non-coplanar case a more complex dependency on the inclination between orbital path and disc plane is found than for distant encounters. For the coplanar prograde case new fitting formulae for the mass and angular momentum loss are obtained, which cover the whole range from grazing to distant encounters. In addition, the final disc size and the mass exchange between discs is examined, demonstrating that for equal mass stars in encounters as close as 1.5 times the disc radius, the disc size only is reduced by approximately $10 \%$.
\end{abstract}

Key words. accretion, accretion disks - methods: $N$-body simulations - methods: numerical

\section{Introduction}

The discovery of planets outside the solar system and detailed observations of protoplanetary discs have been milestones in the understanding of planetary systems; findings which have caused long-standing theories about the formation of planets to be questioned. Currently there are a number of scenarios proposed to explain the formation of planets from the protoplanetary accretion disc surrounding young stars, of which two different schemes - planet formation by the agglomeration of matter in the disc and planet formation induced by gravitational disc instabilities - are the most favoured. In other scenarios, magnetic fields (Balbus \& Hawley 2002; Klahr \& Bodenheimer 2003) are essential. In several of these suggested models, encounters between stars surrounded by their protoplanetary accretion discs play an important role (Bonnell et al. 2001; Kobayashi \& Ida 2001; Bate et al. 2002; Oxley \& Woolfson 2004).

As star-disc systems have a much larger cross-section than stars alone, interactions between two such systems are much more likely than star-star collisions. The discs are influenced by the encountered system even if the periastron $r_{\text {peri }}$ of the encounter is several times larger than the disc size $r_{\text {disc }}$. Although distant encounters are more likely than close encounters, close encounters are not an unlikely event. For example, simulations show that in the ONC cluster around $20 \%$ of all stars undergo encounters closer than $300 \mathrm{AU}$ within $3 \mathrm{Myr}$ (Olczak et al., in preparation).

There have been attempts to treat the problem of the encounter of star-disc systems analytically (Ostriker 1994), but for close encounters the problem becomes highly nonlinear and requires a numerical treatment. In the 1990s several authors investigated the effect on star-disc systems which are disturbed either by an other star or star-discs systems using numerical methods (Heller 1995; Hall et al. 1996; Hall 1997; Larwood 1997; Boffin et al. 1998). At that time the question of binary formation was the driving force behind these investigations. This meant only parabolic encounters were of interest and only a limited number of cases were investigated due to the computational expense of such investigations. Of these studies the work by Hall et al. (1996) spanned the widest parameter range; they treated parabolic encounters of equal mass stars but with a preference of penetrating encounters. More recent investigations (Pfalzner 2003, 2004a) have included hyperbolic encounters but concentrated on coplanar prograde encounters only. A comprehensive data base for all type of disc encounters - whether prograde or retrograde, parabolic or hyperbolic, coplanar or non-coplanar - is still lacking.

This paper provides a first attempt at such a data base, describing the effect of encounters on the global disc features like mass and angular momentum loss, change in disc size etc. for low-mass discs. Due to the computational expense, the parameter space is still limited and an extension will be necessary in the future.

Like in most previous studies, only one of the stars involved in the encounter is surrounded by a disc. Although this was initially done to reduce the complexity and parameter space, for the case of low-mass discs it turns out that the results of star-disc encounters can be relatively easily generalized to discdisc encounters (Pfalzner et al. 2004b), making separate studies 
unnecessary. It should be pointed out that this is not so for highmass discs.

In order to keep the computational effort as small as possible while maximizing the accessible parameter space, in our simulations only the forces of the disc onto the star and vice versa are considered. The effects of pressure, viscosity and self-gravity within the disc are not included. It has been shown by Pfalzner $(2003,2004 a)$ that this is a valid procedure as long as the disc is of low mass and one is only interested in global features. Both conditions are fulfilled here, as the discs chosen to be of mass $m_{\text {disc }}=0.01 M_{\odot}$ to correspond to a value characteristic for most observed discs, and mass and angular momentum transfer are investigated.

A description of the physical set up and the numerical method which is used to simulate the encounters can be found in Sect. 2. From previous studies (Heller 1995; Hall et al. 1996), the strongest impact of the encounter on the disc is expected for prograde coplanar encounters. In Sect. 3 we start with this case and investigate how the mass and angular momentum transfer in the disc depends on the periastron distance and the relative mass of the stars. We show how this transfer also depends on the eccentricity of the encounter paths. The angular momentum change is investigated in two different ways - first we include all particles that are still bound to the star, then we include only the particles still inside the initial disc size. The reason for using these two definitions is the still unanswered question of how the disc matter looses sufficient angular momentum to be accreted onto its star. Although probably collisions will not be the main mechanism for losing angular momentum, there is a certain amount of angular momentum loss due to encounters. As was pointed out by Pfalzner (2004), this angular momentum loss is usually underestimated by using this first definition. However, by considering only particles inside the initial disc size a more physically relevant angular momentum loss is found.

In Sect. 4 a similar investigation for retrograde coplanar encounters is presented. Unless the encounter periastron is very close to the disc "edge", the mass and angular momentum transfer in such retrograde encounters is much smaller than for prograde encounters of the same parameters. It has been argued that prograde encounters are more likely than retrogrades but this is still an open question. The same applies for whether the discs in a cluster are in any way aligned. Although there are some first studies (Jensen et al. 2004) concerning a possible disc alignment, there is no definite answer yet. Therefore in Sect. 5 non-coplanar encounters are studied. It will be shown that even for inclinations of up to approximately 45 degree the mass and angular momentum loss do not differ considerably from the prograde coplanar case. For larger inclinations the effect on the disc is significantly diminished.

In Sect. 6 the disc size after the encounter is studied as function of the encounter parameters. Finally in Sect. 7, it is determined how much disc matter is captured by the passing star.

\section{Disc model and numerics}

In this study both stars are allowed to move freely and only one star (hereafter called star 1) is surrounded by a disc. Since observations indicate that low-mass discs are much more common than high-mass discs, in this study the disc mass is chosen as $m_{\text {disc }}=0.01 M_{\odot}-$ a typical mass value of observed discs. In this study the discs are simulated using 10000 pseudoparticles as tracers of the observed gas. Earlier studies (Pfalzner 2003, 2004; Pfalzner et al. 2004) showed that in the case of such low-mass discs, the effects of pressure, viscous forces and self-gravity on the mass and angular momentum transport in such encounters are negligible. A test particle model therefore suffices, where the disc particles only feel the force of the two stars and vice versa, however going beyond restricted 3-body simulations. Details of the numerical method are described in Pfalzner (2003).

Despite the test model approach, these type of simulations are still computationally expensive, so that higher resolution simulations can only be performed for particular cases. Benchmark simulations using 1 million particles revealed very little quantitative differences to simulations with 10000 particles indicating that the lower resolution suffices to determine the global features we are interested in here.

In all simulations the discs extend initially from 10 to $100 \mathrm{AU}$, the gap of $10 \mathrm{AU}$ exists between the stars and the inner edges of the discs to avoid additional complex calculations of direct star/disc interactions and to save computer time. The density is distributed in the disc according to

$\rho(r, z)=\rho_{0}(r) \exp \left(-\frac{z^{2}}{2 H(r)^{2}}\right)$,

where $H(r)$ is the local vertical half-thickness of the disc and is set constant in this study. $\rho_{0}(r)$ is the mid-plane density with $\rho_{0}(r) \sim 1 / r^{2}$ giving a surface density of $\Sigma \sim 1 / r$, where $\rho_{0}(r)=$ $1.313 \times 10^{-10} \mathrm{~g} / \mathrm{cm}^{3}$ at $r=1$ AU. Star 1 is throughout the study of mass $M_{*}^{1}=1 M_{\odot}$ whereas the mass of star 2 varies in the different encounters between $M_{*}^{2}=0.1 M_{\odot}$ and $2 M_{\odot}$. The periastra of the encounters are chosen between 100 and $450 \mathrm{AU}$. As the discs extends to $r_{\text {disc }}=100 \mathrm{AU}$, this covers the parameter space from grazing to distant encounters.

As described in Pfalzner (2003) the obtained results can be generalized for other mass distribution within the disc by applying appropriate scaling factors.

\section{Prograde coplanar encounters}

In this study we exclude the case where the encounter leads to the formation of a bound system. Then of all possible encounter paths, parabolic encounters lead to the longest interaction time and have the strongest impact on the disc. We will start our investigation by studying the effects of such parabolic prograde encounters. In this case the parameters which characterize the encounters are the masses of the stars, $M_{1}^{*}$ and $M_{2}^{*}$, and the periastron $r_{\text {peri }}$. In this study we choose to keep the mass of star 1 constant at $M_{1}^{*}=1 M_{\odot}$ and vary $M_{2}^{*}$ and $r_{\text {peri }}$.

In Fig. 1 the dependence of the mass loss $\Delta m_{\text {disc }} / m_{\text {disc }}$ and angular momentum loss $\Delta J / J$ in the disc are shown as a function of $r_{\text {peri }} / r_{\text {disc }}$. As expected, the mass and angular momentum transport decreases for larger periastra (see Fig. 1). For the equal mass case $M_{1}^{*}=M_{2}^{*}=1 M_{\odot}$ the mass loss is approximately $48 \%$ and angular momentum loss $(\Delta J / J)_{\text {total }}$ 
a)

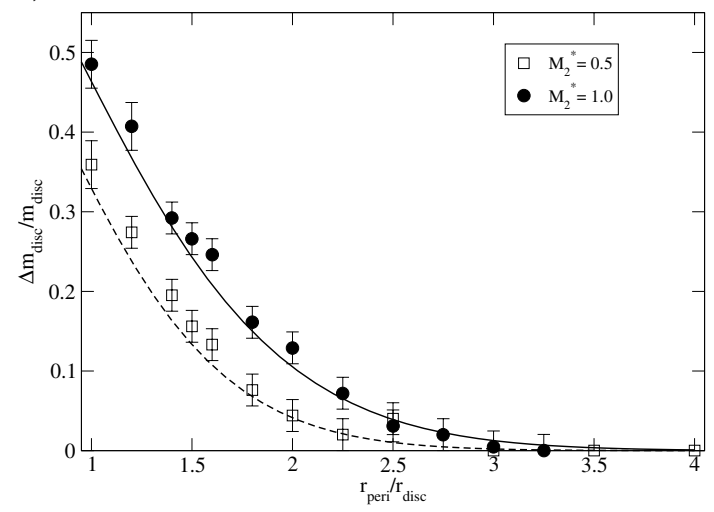

b)

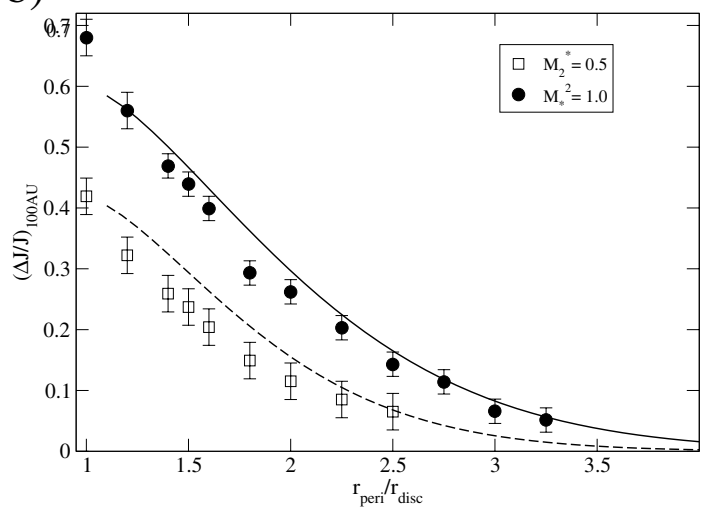

c)

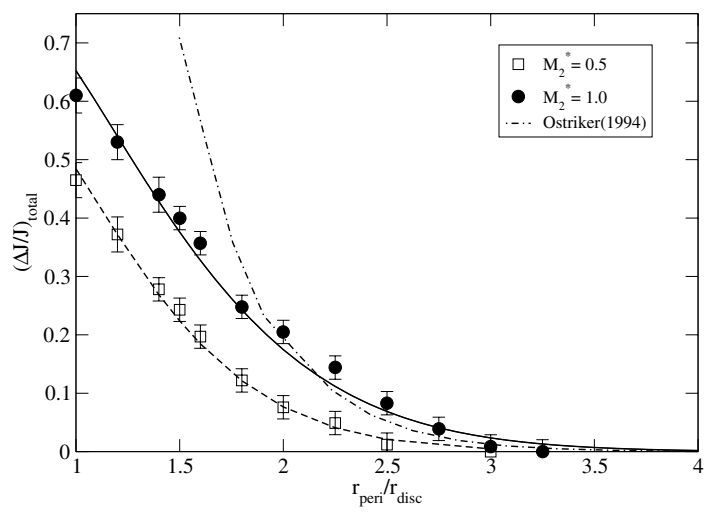

Fig. 1. Prograde encounters: relative a) mass loss and b) angular momentum loss within $100 \mathrm{AU}$ and $\mathbf{c}$ ) in total as a function of the periastron from the primary star. The periastron is given in units of the disc size, in the here considered case that was $100 \mathrm{AU} . M_{2}^{*}$ is given in units of solar masses. The analytical result of the total angular momentum loss by Ostriker (1994) is indicated in c).

about $62 \%$ of the initial values for the closest investigated case of $\left(r_{\text {peri }} / r_{\text {disc }}=1\right)$. For a lower mass of star $2\left(M_{2}^{*}=0.5 M_{\odot}\right)$ the mass loss is still $36 \%$ and total angular momentum about $46 \%$. For both cases an exponential decrease of the mass and angular momentum loss with $r_{\text {peri }} / r_{\text {disc }}$ is found.
For distant encounters Ostriker (1994) derived the following formula for the angular momentum loss,

$$
\begin{array}{r}
\Delta J \sim \frac{M_{2}^{*}}{M_{1}^{2}+M_{2}^{*}} \exp \left[-\sqrt{\left.\frac{M_{1}^{*}}{M_{1}^{2}+M_{2}^{*}}\left(\frac{r_{\text {peri }}}{r_{\text {disc }}}\right)^{3 / 2}\right]}\right. \\
\times \frac{2}{\Omega\left(r_{\text {disc }}\right)} \cos \left(\frac{\beta}{2}\right)^{5},
\end{array}
$$

where $\Omega\left(r_{\text {disc }}\right)$ is the angular velocity at the outer disc radius and $\beta$ the inclination between the disc plane and the orbital plane. In the coplanar case considered here, $\beta=0$. A comparison of this analytical result and our simulation data (see Fig. 1c) shows good agreement for distant encounters, but for encounters closer than $r_{\text {peri }} / r_{\text {disc }}<2$ (in the case $M_{2}^{*}=1 M_{\odot}$ ) the analytical results considerably overestimate the angular momentum loss.

Due to the encounter a portion of the disc particles are only loosely bound to the disc, undergoing eccentric orbits far outside the original disc size. These particles contribute to the total angular momentum but are irrelevant to the angular momentum loss connected to accretion. Therefore in Fig. 1 and all following figures that concern the angular momentum loss, the total angular momentum loss $(\Delta J / J)_{\text {total, }}$, which includes all particles bound to star 1 , as well as the angular momentum loss $(\Delta J / J)_{100}$ of the particles bound within $100 \mathrm{AU}$ are shown. This is done to obtain a better measure of the the angular momentum loss inside the disc (Pfalzner 2004) relevant for the accretion process. As some of the bound particles move on strongly elliptical orbits, they leave and reenter the $100 \mathrm{AU}$ range periodically. This leads to a periodic change of $J_{100}$, but this change is in the considered parameter range below $1 \%$, so that it can be neglected.

As we can see already in Fig. $1 \mathrm{~b}$ for certain encounter parameters $(\Delta J / J)_{100}$ can be considerably larger than $(\Delta J / J)_{\text {total }}$. $(\Delta J / J)_{100}$ decreases less steeply than $(\Delta J / J)_{\text {total }}$ for larger $r_{\text {peri }} / r_{\text {disc }}$. In other words: for close encounters there is little difference between $(\Delta J / J)_{100}$ and $(\Delta J / J)_{\text {total }}$ whereas it can be considerable for distant encounters. For example, for $M_{2}^{*}=1 M_{\odot}$ and $r_{\text {peri }} / r_{\text {disc }}=2.75,(\Delta J / J)_{100}$ is more than twice $(\Delta J / J)_{\text {total }}$. Since distant encounters are actually much more likely to occur than close ones, this might be crucial for the loss of angular momentum to the star over time through repeated distant encounters and might actually facilitate the accretion of matter from the disc onto the star.

Figure 2 shows how the mass and angular momentum loss increases with the mass $M_{2}^{*}$ of star 2. The mass and angular momentum loss are shown for different periastra, $r_{\text {peri }}=100 \mathrm{AU}$, $150 \mathrm{AU}$ and $200 \mathrm{AU}$, which corresponds to $r_{\text {peri }} / r_{\text {disc }}=1$, 1.5 and 2 in Fig. 1, respectively. For small masses of $M_{2}^{*}$, i.e. $M_{2}^{*}=0.1$, the losses in mass and angular momentum are all below $10 \%$ for all shown periastra, apart from $(\Delta J / J)_{100}$ at $100 \mathrm{AU}$. However, for higher masses $M_{2}^{*}$ the effect on the disc rises steeply but might eventually approach a certain maximum level for high masses $M_{2}^{*}$. For a periastron of $100 \mathrm{AU}$ $\Delta m_{\text {disc }} / m_{\text {disc }}$ and $(\Delta J / J)_{\text {total }}$ are around $55 \%$, for $r_{\text {peri }}=150 \mathrm{AU}$ they are about $35 \%$ and for $r_{\text {peri }}=150$ AU still about $25 \%$. So that for a close encounter with low mass stars only a small effect is expected, whereas a close encounter with a roughly 
a)

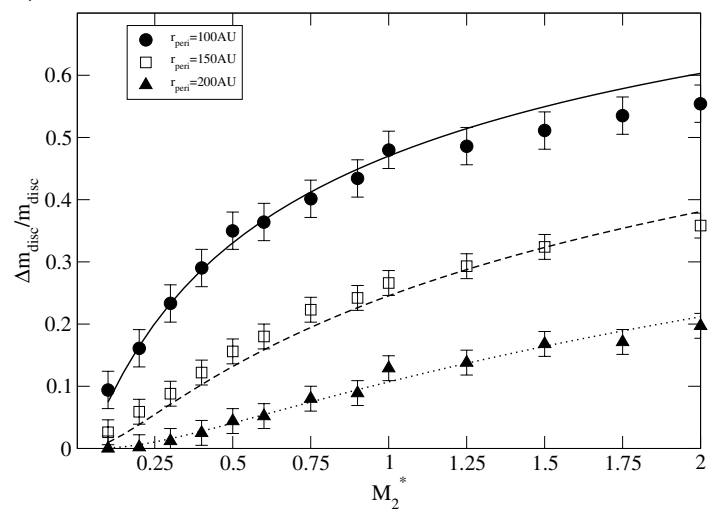

b)

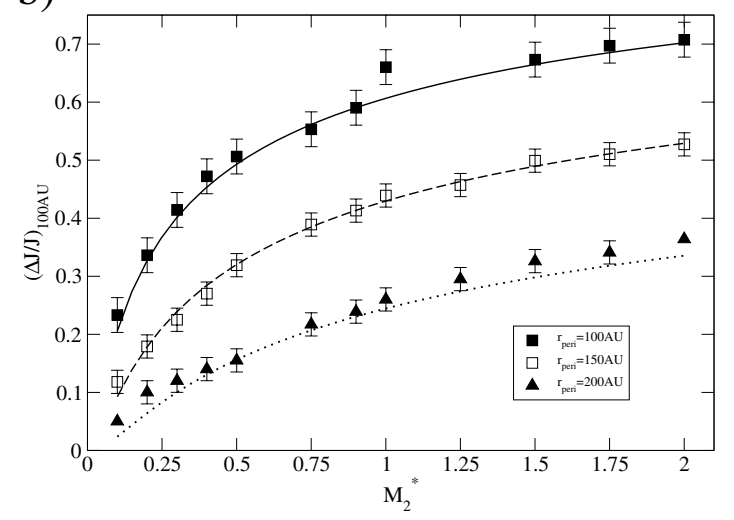

c)

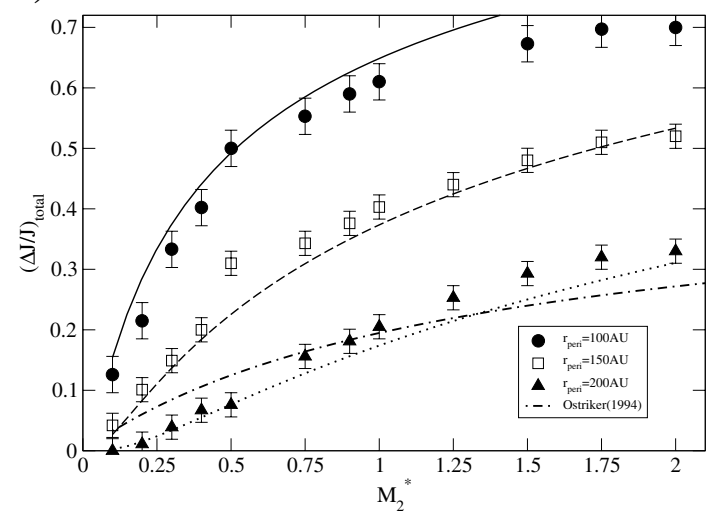

Fig. 2. Prograde encounters: relative a) mass loss and b) angular momentum loss b) within $100 \mathrm{AU}$ and $\mathbf{c}$ ) in total as a function of the mass $M_{2}^{*}$ (in units of solar masses) of star 2 for encounters with periastra of 100, 150 and $200 \mathrm{AU}$. In c) the result of Ostriker (1994) is shown in comparison for the case $r_{\text {peri }} / r_{\text {disc }}=200 \mathrm{AU}$.

equal mass star leads to the loss of a quarter to half the disc mass.

The results in Figs. 1 and 2 can be fitted by the following formula

$$
\frac{\Delta X}{X}=A \exp \left[-\frac{1}{2} \sqrt{\frac{M_{1}^{*}}{M_{2}^{*}}}\right] \exp \left[-\sqrt{\frac{M_{1}^{*}}{M_{2}^{*}}}\left(\frac{r_{\text {peri }}-r_{\text {in }}}{r_{\text {disc }}}\right)^{3 / 2}\right] .
$$

For our results of the mass loss $\Delta X / X=\Delta m_{\text {disc }} / m_{\text {disc }}$ best fitting is obtained with the parameters $A$ and $r_{\text {in }}$ in Eq. (3) being set $A=1.1$ and $r_{\text {in }}=40$ AU. Whereas for the total angular momentum loss $\Delta X / X=(\Delta J / J)_{\text {total }}$ the data require $A=1.26$ and $r_{\text {in }}=70 \mathrm{AU}$.

Equation (3) shows some similarities to the dependencies in the analytical result of the total angular momentum loss for distant encounters by Ostriker(1994). Comparing Eq. (3) with the Ostriker result given by Eq. (1) it can be seen that for the limit $r_{\text {peri }} \longrightarrow \infty$ the dependence on the periastron distance is recovered. However, the mass dependence differs. Ostriker derived Eq. (1) under the assumption of a small perturbation i.e. large periastron or small perturber mass. For $M_{2}^{*}>1 M_{\odot}$ a deviation from the analytical result is therfore not unexpected. For $M_{2}^{*}<1 M_{\odot}$ the deviation in the results are probably due to the large statistical errors in the simulation results.

The angular momentum loss within $100 \mathrm{AU}$ needs a slightly more complex fitting formula

$$
\begin{aligned}
\left(\frac{\Delta J}{J}\right)_{100}= & A \exp \left[-\frac{1}{2} \sqrt{\frac{M_{1}^{*}}{M_{2}^{*}}}\right] \\
& \times \exp \left[-\sqrt{\frac{M_{1}^{*}}{M_{2}^{*}}\left(\frac{0.7\left(r_{\text {peri }}-r_{\text {in }}\right)}{r_{\text {disc }}}\right)^{3}}\right] .
\end{aligned}
$$

Here $r_{\text {in }}=100 \mathrm{AU}$ but $A$ is different for each periastron, i.e. $A=1$ for $r_{\text {peri }}=100 \mathrm{AU}, A=0.85$ for $r_{\text {peri }}=150 \mathrm{AU}, A=$ 0.72 for $r_{\text {peri }}=200 \mathrm{AU}$.

Previous work (Pfalzner 2004a) suggests that in terms of mass and angular momentum transfer, hyperbolic encounters are "failed" parabolic encounters. The interaction time in hyperbolic encounters is too short to enable the complete mass and angular momentum transfer that one would have in a parabolic encounter. Figure 3 supports this view. It shows the mass and angular momentum loss as a function of the eccentricity of the encounter for $M_{2}^{*}=0.5$ and $M_{2}^{*}=1.0$. The mass and angular momentum transfer values for the parabolic encounter with $r_{\text {peri }} / r_{\text {disc }}=100 \mathrm{AU}$ is taken as reference and is therefore 1. Figure 3 shows how the mass and angular momentum loss in the encounter with the same mass of star 2 and periastron becomes less efficient as the path becomes increasingly eccentric. So for an eccentricity $\epsilon=10$ the mass and total angular momentum transfer is only around $20 \%$ of that in a parabolic encounter. As for the dependence on the periastron and the mass of the secondary star here again $(\Delta J / J)_{100}$ decreases less rapidly than $(\Delta J / J)_{\text {total }}$ and $\Delta m_{\text {disc }} / m_{\text {disc }}$ with increased eccentricity.

The decrease in disc mass loss with increasing ellipticity seems proceed in the same way for $M_{2}^{*}=1 M_{\odot}$ as for $M_{2}^{*}=0.5 M_{\odot}$. As both data point sets can be fitted very well with an exponential decrease of $\exp (-0.4(\epsilon-1)) / \epsilon^{0.5}$. However, it should be noted that although the shape of the curve for $(\Delta J / J)_{100}$ and $(\Delta J / J)_{\text {total }}$ is for both masses very similar the $M_{2}^{*}=0.5$ values are always a bit less than $M_{2}^{*}=1.0$ values.

A mention about the errors is in order. There are several sources of error; a) the limited resolution of the disc, b) deviations from the ideal parabolic path and c) for close encounters the actual particle distribution at the "edge" of the disc might play a role. Comparing the results of different simulations with the same interaction parameters, we conclude that for prograde 
a)

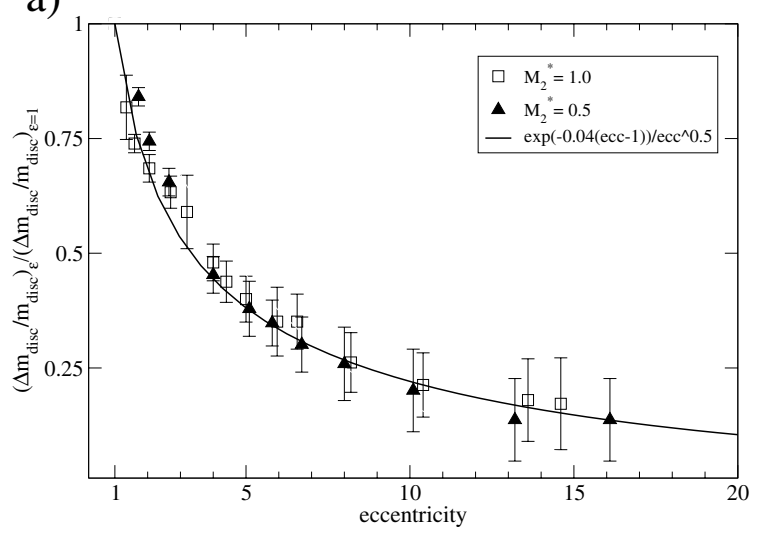

b)

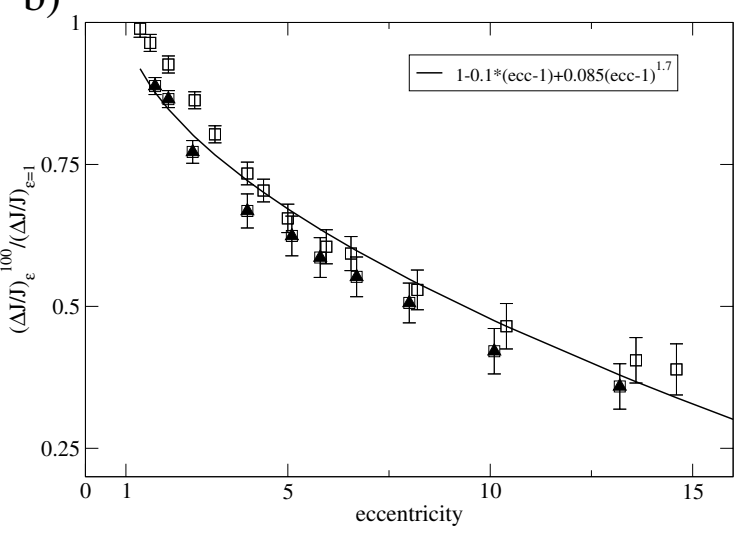

c)

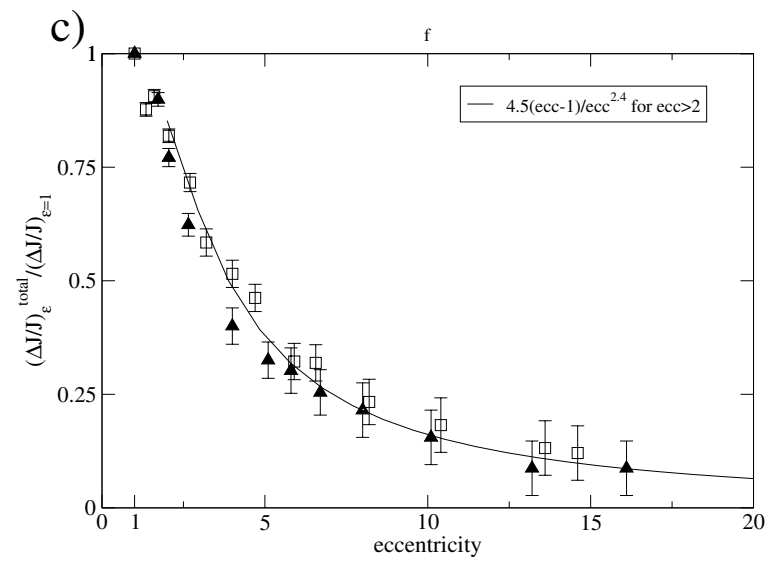

Fig. 3. Prograde encounters: relative a) mass loss and b) angular momentum loss within $100 \mathrm{AU}$ and $\mathbf{c}$ ) in total as a function of the eccentricity of the orbit of star 2. The values for the parabolic encounter are chosen as points of reference $(=1)$.

encounters the absolute error of the mass and angular momentum loss is about $2-3 \%$.

\section{Retrograde coplanar encounters}

Retrograde encounters have been investigated to a much lesser degree than prograde encounters. There are two reasons for this, first, the few previous results (Heller 1995; Hall 1997) indicate that retrograde encounters change the mass and angular momentum distribution in the disc far less, second, it has been argued that prograde encounters are more likely to occur than retrograde encounters. The second reason is based on the assumption that star-disc systems that encounter each other are likely to have a common formation history. If this is the case, there could exist a preference for the discs of having the same rotational orientation as well. Obviously, this argument is strongly linked to the underlying star formation picture. To our knowledge there exists no observational evidence that would suggest a preference of prograde orientations of neighbouring disc systems. As long as this point is not clarified, the same information about the effect of encounters on the discs is needed for retrograde encounters as for the prograde case.

In the analytical result of Ostriker in Eq. (1) the retrograde case is equivalent to $\beta=\pi$. In this case Eq. (1) predicts that there is no angular momentum loss. Although this is valid for distant encounters, our simulation results show that this is not true for close encounters.

Figure 4 illustrates the dependence of the mass and angular momentum loss in the disc as function of $r_{\text {peri }} / r_{\text {disc }}$ for the retrograde case in parabolic encounters. In agreement with previous results (Heller 1995; Hall 1997) there is no mass and total angular momentum loss for encounters more distant than $r_{\text {peri }} / r_{\text {disc }}=1.5$. It can be seen that even in the periastron range of $1.25<r_{\text {peri }} / r_{\text {disc }}<1.5$ the change in mass and total angular momentum is always less than $3 \%$. However, there is a steep increase in $(\Delta J / J)_{\text {total }}$ and $\Delta m_{\text {disc }} / m_{\text {disc }}$ as the encounters become so close that the passing star nearly penetrates the disc. For example, in the case $M_{2}^{*}=1.0$ and $r_{\text {peri }} / r_{\text {disc }}=1$, the mass as well as $(\Delta J / J)_{\text {total }}$ is about $13-14 \%$. However, even in these cases the mass and angular momentum loss in retrograde encounters are about a factor 3 less than for the same prograde encounter.

Here again $(\Delta J / J)_{100}$ is a much less steep function of $r_{\text {peri }} / r_{\text {disc }}$ than $(\Delta J / J)_{\text {total }}$ and $\Delta m_{\text {disc }} / m_{\text {disc }}$. Interestingly so that for example at $r_{\text {peri }} / r_{\text {disc }}=1.5$ and $M_{2}^{*}=1 M_{\odot}$ there is still about $8 \%$ loss of $(\Delta J / J)_{\text {total }}$, but non for the mass or the total angular momentum.

Figure 5 shows how the mass and the total angular momentum loss depends on $M_{2}^{*}$ for a retrograde encounter. As only for very close encounters considerable mass and angular momentum transport can be expected, the cases $r_{\text {peri }}=100 \mathrm{AU}$ and $r_{\text {peri }}=110 \mathrm{AU}$ were investigated. The shape of the curves is similar to the prograde case shown in Fig. 2, which points to a similar depends on $M_{2}^{*}$ as in the prograde encounter. However, the values of $\Delta m_{\mathrm{disc}} / m_{\mathrm{disc}}$ and $\Delta J / J$ are much less. For example, is $\Delta m_{\text {disc }} / m_{\text {disc }}$ for $r_{\text {peri }} / r_{\text {disc }}=1.0$ and $M_{2}^{*}=1.5 M_{\odot}$ for this retrograde encounter less than half of that in the prograde encounter.

How do $\Delta m_{\text {disc }} / m_{\text {disc }}$ and $\Delta J / J$ depend on the eccentricity of the encounter in the retrograde case? Figure 6 shows that the mass and angular momentum loss is much less even if the encounter is only slightly hyperbolic. So even slight deviations from the parabolic case seem to affect retrograde encounters more than parabolic encounters. This means there is a larger error to be expected for the retrograde data than for the prograde results. 
a)

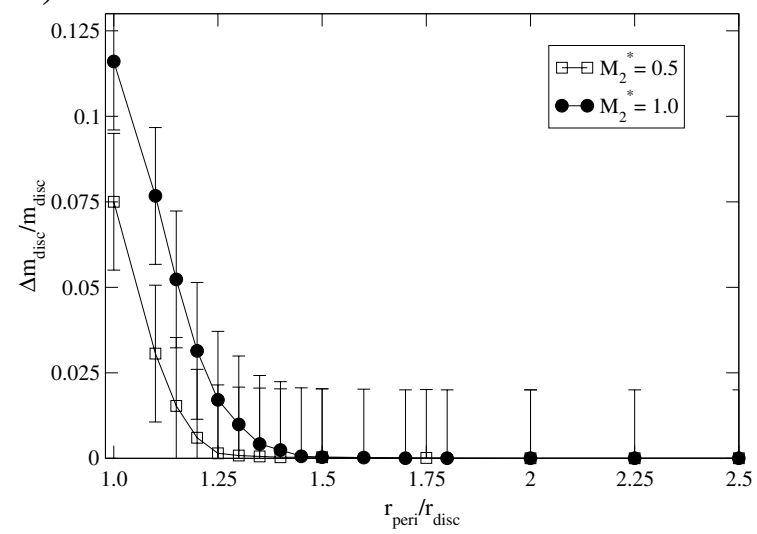

b)

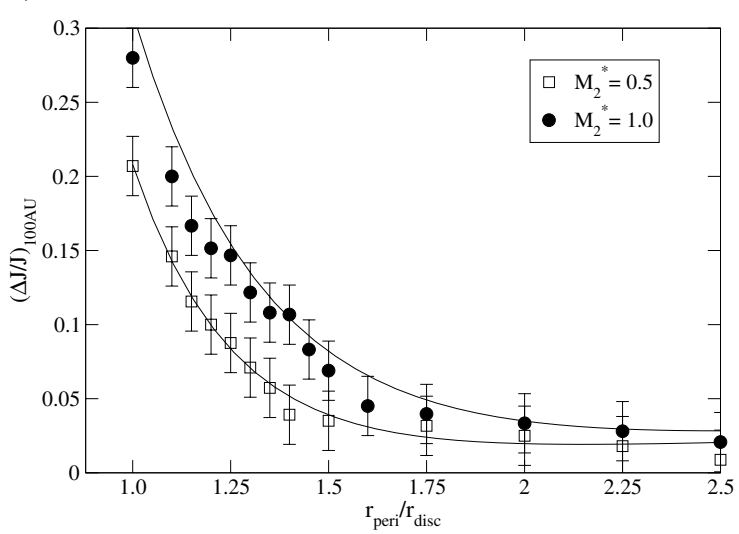

c)

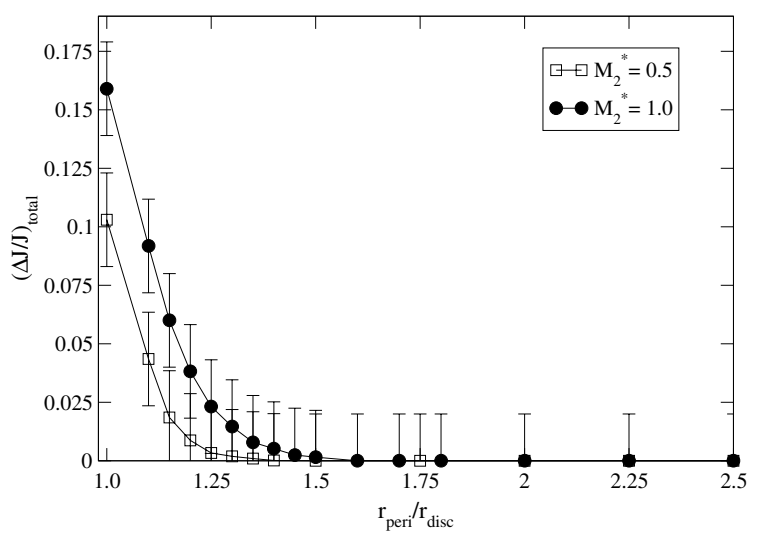

Fig. 4. Retrograde encounters: relative a) mass loss and b) angular momentum loss within $100 \mathrm{AU}$ and $\mathbf{c}$ ) in total as a function of the periastron from the primary star. The periastron is given in units of the disc size, in the here considered case that was $100 \mathrm{AU} . M_{2}^{*}$ is given in units of solar masses.

Apart from the prograde parabolic nearly penetrating case, retrograde encounters cause no mass transfer or changes to $J_{\text {total }}$ occur. Only $J_{100}$ seems to be effected.

\section{Non-coplanar encounters}

What happens if the encounter is not coplanar? From previous studies it is known that although non-coplanar encounters
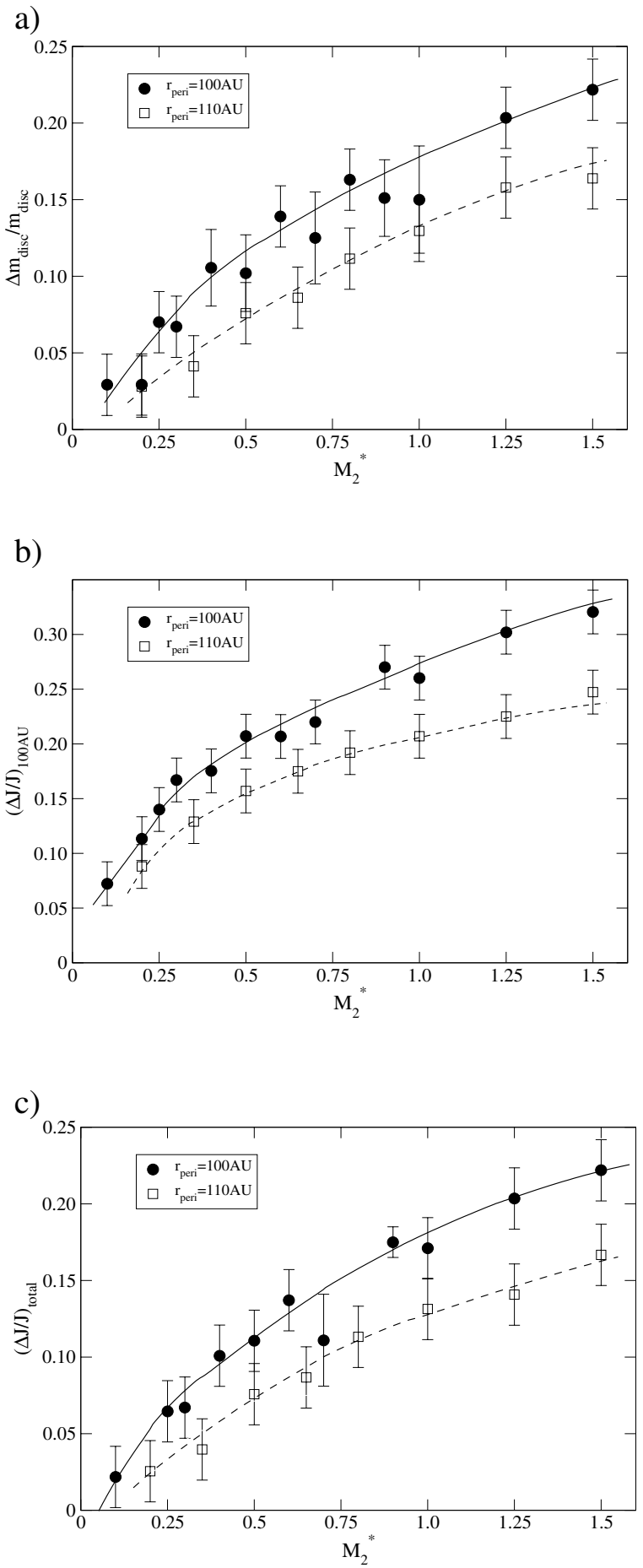

Fig. 5. Retrograde encounters: relative a) mass loss and b) angular momentum loss b) within $100 \mathrm{AU}$ and $\mathbf{c}$ ) in total as a function of the mass $M_{2}^{*}$ of the secondary star for encounters with periastra of 100 and $110 \mathrm{AU} . M_{2}^{*}$ is given in units of solar masses.

can lead to warping etc., less mass and angular momentum are lost than in prograde coplanar encounters. Here we attempt to quantify the effect of non-coplanarity. We investigate 4 different cases given by parameter sets $r_{\text {peri }} / r_{\text {disc }}$ and $M_{2}^{*}$, varying the angle of inclination between the plane of the path of star 2 and the disc orientation. The result is shown in Fig. 7. Here the coplanar prograde encounter corresponds to an angle of 
a)
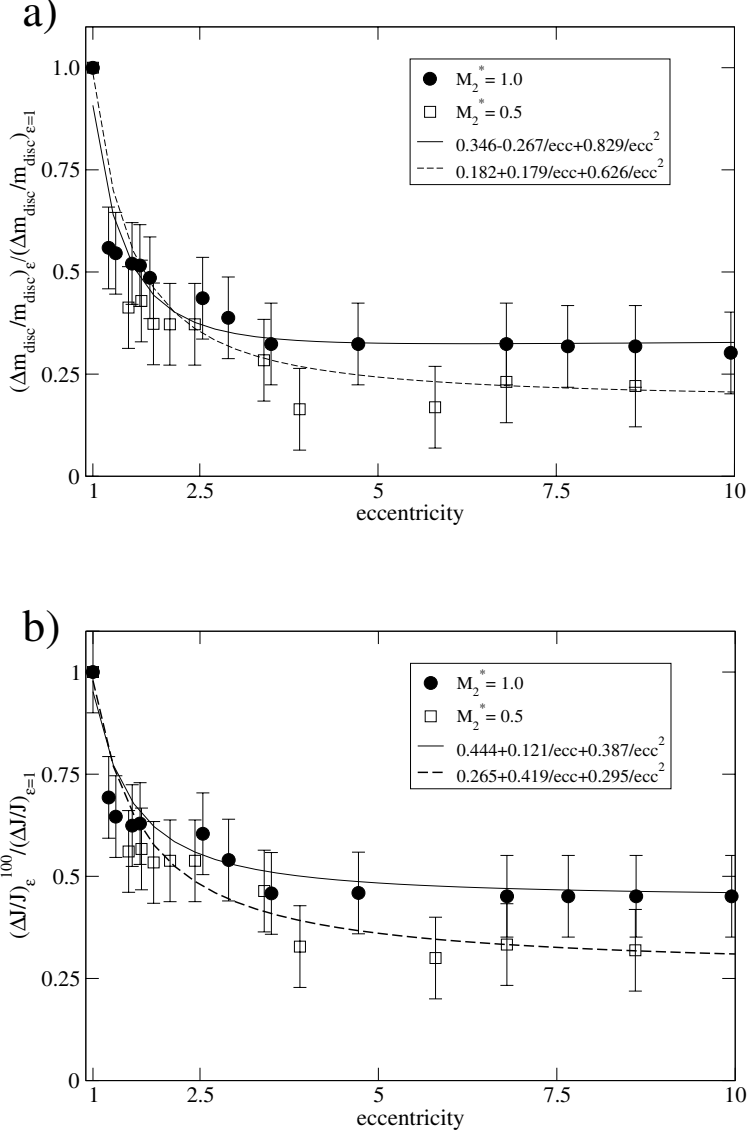

c)

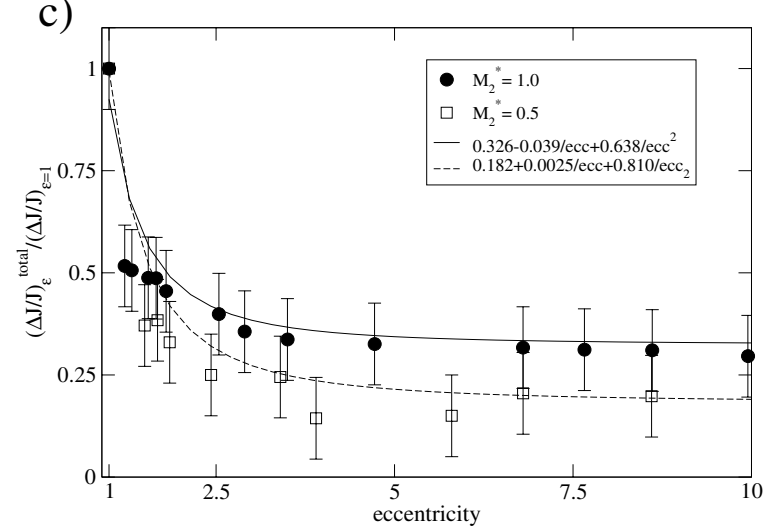

Fig. 6. Retrograde encounters: relative a) mass loss and b) angular momentum loss b) within $100 \mathrm{AU}$ and $\mathbf{c}$ ) in total as a function of the eccentricity of the encounter. $M_{2}^{*}$ is given in units of solar masses.

0 degree and the coplanar retrograde encounter to an angle of 180 degree.

Figure 7 shows that there is the general trend for all 4 cases that a slight deviation from coplanarity does not effect $\Delta m_{\mathrm{disc}} / m_{\mathrm{disc}}$ or $\Delta J / J$ very much, only for inclinations larger than 45 degree this changes.

We now look at the 4 different sets separately. These 4 parameter sets were chosen in such a way that they represent different typical situations. It is necessary to investigate a distant encounter to be able to compare with the analytical treatment of a)

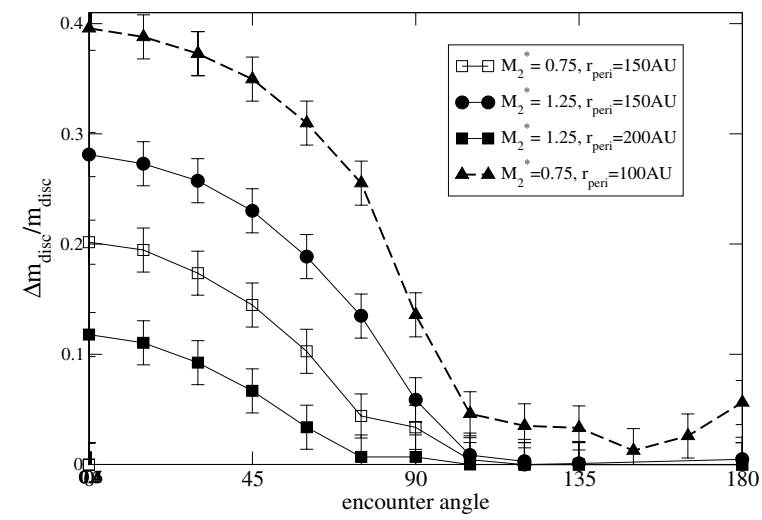

b)

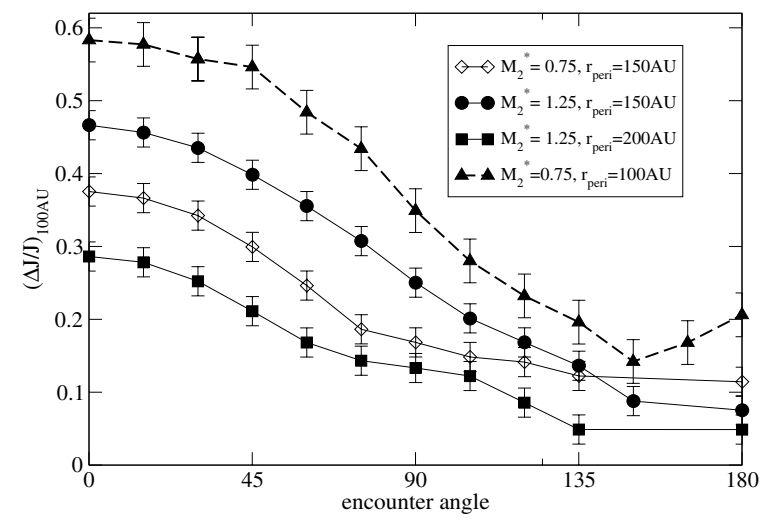

c)

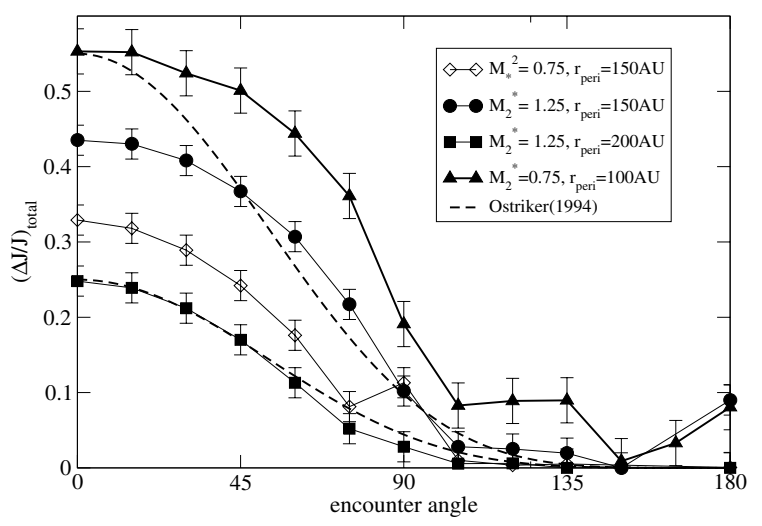

Fig. 7. Non-coplanar encounters: relative a) mass loss and b) angular momentum loss b) within $100 \mathrm{AU}$ and $\mathbf{c}$ ) in total as a function of the mass $M_{2}^{*}$ of the secondary star for encounters with periastra of 100 , 150 and $200 \mathrm{AU}$. The analytical result of the total angular momentum loss by Ostriker (1994) is indicated in c) for most distant encounter with $r_{\text {peri }}=200 \mathrm{AU}$ and the strongest encounter with $r_{\text {peri }}=100 \mathrm{AU}$ and $M_{2}^{*}=0.75 M_{\odot}$.

distant encounters by Ostriker (1994). The case $M_{2}^{*}=1.25 M_{\odot}$ and $r_{\text {peri }} / r_{\text {disc }}=2$ represents such a distant encounter, where the relatively high mass of star 2 ensures that in the prograde coplanar case the losses are still larger than a few percent to 
be able to clearly distinguish them from the errors which are typically in the $2-3 \%$ range.

The corresponding retrograde encounter leads to no mass or angular momentum transfer. Figure 7 shows that there is a smooth transition from the coplanar encounter value to this retrograde value. For more than approximately 100-120 degree of inclination there is no more change in $\Delta m_{\mathrm{disc}} / m_{\mathrm{disc}}$ or $(\Delta J / J)_{\text {total }}$. For $(\Delta J / J)_{100}$ there is some change at all angles, which is nevertheless highest for coplanar encounters.

The case $M_{2}^{*}=0.75$ and $r_{\text {peri }}=150$ AU shows, although a closer encounter, more or less the same dependence on the relative orientation of the path and the disc. Only the absolute values for $\Delta m_{\text {disc }} / m_{\text {disc }}$ and $\Delta J / J$ are larger.

The situation is different for $M_{2}^{*}=1.25$ and $r_{\text {peri }}=200 \mathrm{AU}$, here in the retrograde encounter, mass and angular momentum are lost as well. The data seem to indicate that the minimum of mass and angular momentum transfer is not for the retrograde coplanar case but somewhere between 120 and 180 degrees. As the loss values are very small, this could be just a statistical error.

However, the case $M_{2}^{*}=1.25 M_{\odot}$ and $r_{\text {peri }}=100 \mathrm{AU}$ leaves no room for doubt. Here the retrograde value is well out of this error range and here again the smallest transfer of mass and angular momentum lies between 120 and 165 degree. So that the least mass and angular momentum transfer can be expected for slightly non-coplanar retrograde encounters.

In Fig. 7c we test whether in the non-coplanar case $(\Delta J / J)_{\text {total }}$ shows the $\cos (\beta / 2)^{5}$-dependence of the Ostriker result (Eq. (1)) on the inclination between the orbital and the disc plane. This was done for the two cases $r_{\text {peri }} / r_{\text {disc }}=2$, $M_{2}^{*}=1.25$ and $r_{\text {peri }} / r_{\text {disc }}=1, M_{2}^{*}=0.75$. In the more distant case the simulation results agree reasonable well with this $\cos (\beta / 2)^{5}$-dependence. However, for closer encounters the dependence on the inclination angle is much more complex.

\section{Disc mass transport between stars}

In encounters mass can not only be lost from the disc and drift away from star 1, but star 2 can also capture disc matter. An interesting situation arises if one studies encounters where both stars are surrounded by discs. Then the mass transported between the discs can lead to chemical mixing of the two disc components. Pfalzner et al. (2005) showed that the results for star-disc encounters can be directly applied to disc-disc encounters, as long as the disc masses are low. In the following therefore this capturing of disc material by star 2 will be investigated as well with the chemical mixing in disc-disc encounters in mind.

From our simulations we find that such mass capture of disc material nearly exclusively happens for prograde encounters. The only exception is if star 2 nearly or actually penetrates the disc. So in the following we restrict the investigation to prograde, coplanar parabolic encounters again.

In Fig. 8 the relative disc mass captured by star 2 in such prograde, coplanar parabolic encounters is shown. It can be seen how the captured mass depends on a) the mass $M_{2}^{*}$ of star 2 and b) the relative periastron distance $r_{\text {peri }} / r_{\text {disc }}$ in the
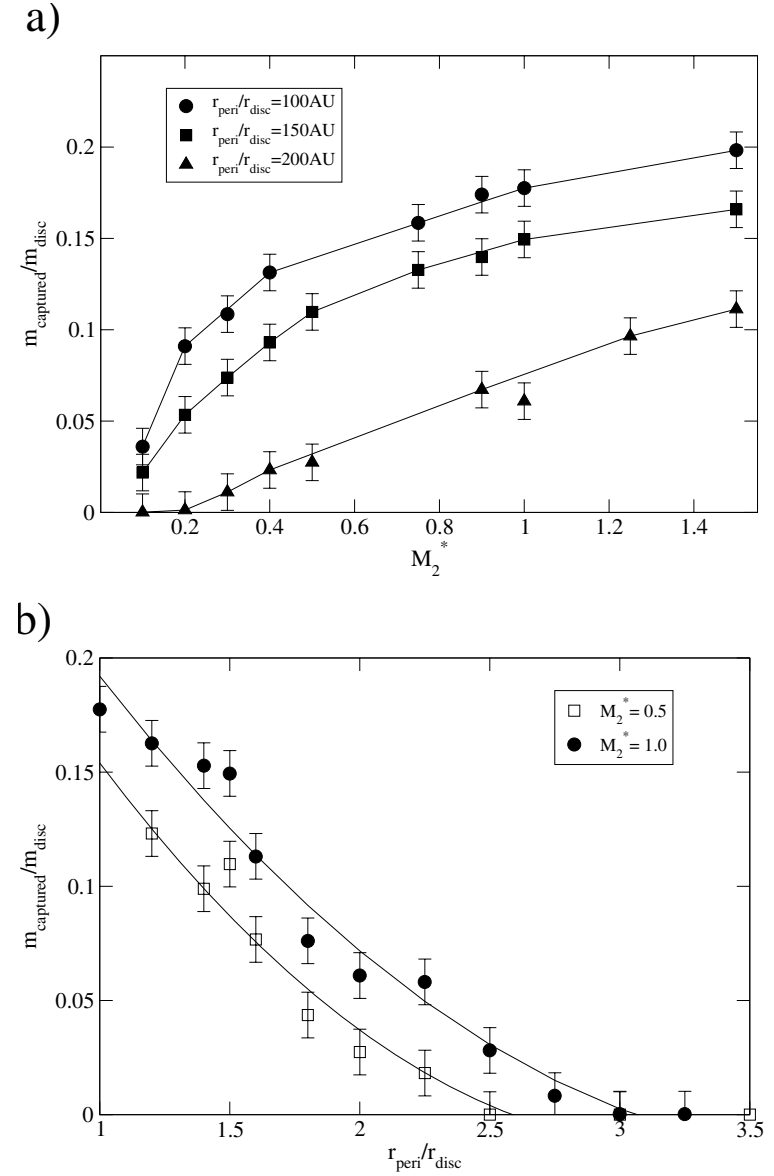

Fig. 8. Relative mass captured from the disc of star 1 by star 2 as function of a) the mass of star 2, b) the relative periastron $r_{\text {peri }} / r_{\text {disc }}$. In a) the simulations were performed for $r_{\text {peri }}=100 \mathrm{AU}, 150 \mathrm{AU}$ and $200 \mathrm{AU}$. In b) the cases of $M_{2}^{*}=0.5 M_{\odot}$ and $M_{2}^{*}=1 M_{\odot}$ were investigated. $M_{2}^{*}$ is given in units of solar masses.

encounter. For low masses of $\operatorname{star} 2\left(M_{2}^{*}=0.1 M_{\odot}\right)$ the captured mass is below $5 \%$ of the disc mass even for grazing encounters with $r_{\text {peri }}=100$ AU. However, for higher masses of star $2\left(M_{2}^{*}=1.5 M_{\odot}\right)$ up to $20 \%$ of the mass of the disc around star 1 can be captured by star 2 . For close encounters $\left(r_{\text {peri }} / r_{\text {disc }}=1.0\right.$ and 1.5) the captured mass is a steep function of the mass of star 2 as long as $M_{2}^{*}<0.5 M_{\odot}$, for higher masses $\left(M_{2}^{*}>0.8 M_{\odot}\right)$ the captured mass seems to depend much less strongly on $M_{2}^{*}$. The curves in Fig. 8a become rather flat for $M_{2}^{*}>0.8 M_{\odot}$ indicating that there is possibly an upper limit for the mass transfer to the second star for each periastron distance.

Figure $8 \mathrm{~b}$ shows the dependence of the captured mass on $r_{\text {peri }} / r_{\text {disc }}$ for $M_{2}^{*}=0.5 M_{\odot}$ and $M_{2}^{*}=1 M_{\odot}$. The decrease in captured mass for larger $r_{\text {peri }} / r_{\text {disc }}$ seems to depend nearly linear on the periastron distance, with a similar slope for $M_{2}^{*}=$ $0.5 M_{\odot}$ and $M_{2}^{*}=1 M_{\odot}$. For encounters more distant than $200 \mathrm{AU}$ the captured mass is well below $10 \%$.

\section{Disc size}

The typical effect in such encounter is that some mass is lost from the outside of the discs as well as some mass transported 
inwards. The density profile in the disc is changed and due to the relative low density at the outside of the disc the whole disc will appear smaller.

An important question for the likelihood of the formation of planetary systems is the survival rate of discs in a cluster. The collision rate as well as the effect of an encounter on the disc size are important parameters in this context. Here we want to investigate the latter and see how the disc size after the encounter depends on the encounter parameters. The study will be limited to prograde coplanar, parabolic encounters for the already above described reason that retrograde encounters influence the disc to a much lesser degree. In addition in the case of non-coplanar encounters, discs become warped which would make the definition of disc size more complex.

In Sect. 2 it was shown that the initial disc has a sharp cutoff at 100 AU. In reality such a sharp cut-off does not exist but a smooth transition towards lower disc densities for larger distances. In the following the disc size is not defined by this $100 \mathrm{AU}$ limit, but as the radius were $95 \%$ of the disc mass are enclosed. For our disc that is at 92 AU.

To demonstrate this effect of an encounter Fig. 9 shows the relative disc size (with the unperturbed disc as reference value 1) after encounters with different parameters. Figure 9a demonstrates that the disc size remains relative unaltered for distant encounters (here indicated by the black triangle for the $r_{\text {peri }}=200 \mathrm{AU}$ data). Even for relatively high masses of star 2 $\left(M_{2}^{*}=1.5 M_{\odot}\right)$ the disc size is still $90 \%$ of the initial disc size.

However, for grazing encounters (indicated by $\bullet$ for $r_{\text {peri }}=$ $100 \mathrm{AU}$ ) the disc size can be reduced by up to $40 \%$, if the mass of star 2 is large enough $\left(M_{2}^{*}=1.5 M_{\odot}\right)$. However, if star 2 is of much lower mass than star $1\left(M_{*}^{2}<0.4 M_{*}^{1}\right)$, even in grazing encounters the disc size is not reduced to less than $90 \%$ of its original size.

Figure $9 \mathrm{~b}$ supports these finding. Here the relative disc size is shown as a function of $r_{\text {peri }} / r_{\text {disc }}$. Again it can be seen that the disc size is reduced by less than $10 \%$ for distant encounters $\left(r_{\text {peri }} / r_{\text {disc }}>2\right)$. Only for closer encounters the disc size is considerably reduced.

From these considerations it can be concluded that relatively close encounters with a high mass of star 2 are necessary to really reduce the disc size. The effect on the disc is much more an alteration of mass distribution profile than the disc radius as such.

\section{Summary}

In this paper we presented a first parameter study of the effect of encounters between two stars where one is surrounded by a low-mass protoplanetary accretion disc for all major types of encounters, i.e. prograde/retrograde; coplanar/non-coplanar and parabolic/hyperbolic. The mass loss of the disc, its angular momentum change, the resulting disc size and the amount of matter captured by the star were investigated.

As indicated by the special cases studied in previous work, this parameter study confirms that coplanar, prograde parabolic encounters lead to the highest mass and angular momentum loss; for example nearly $50 \%$ of the initial disc mass and a)
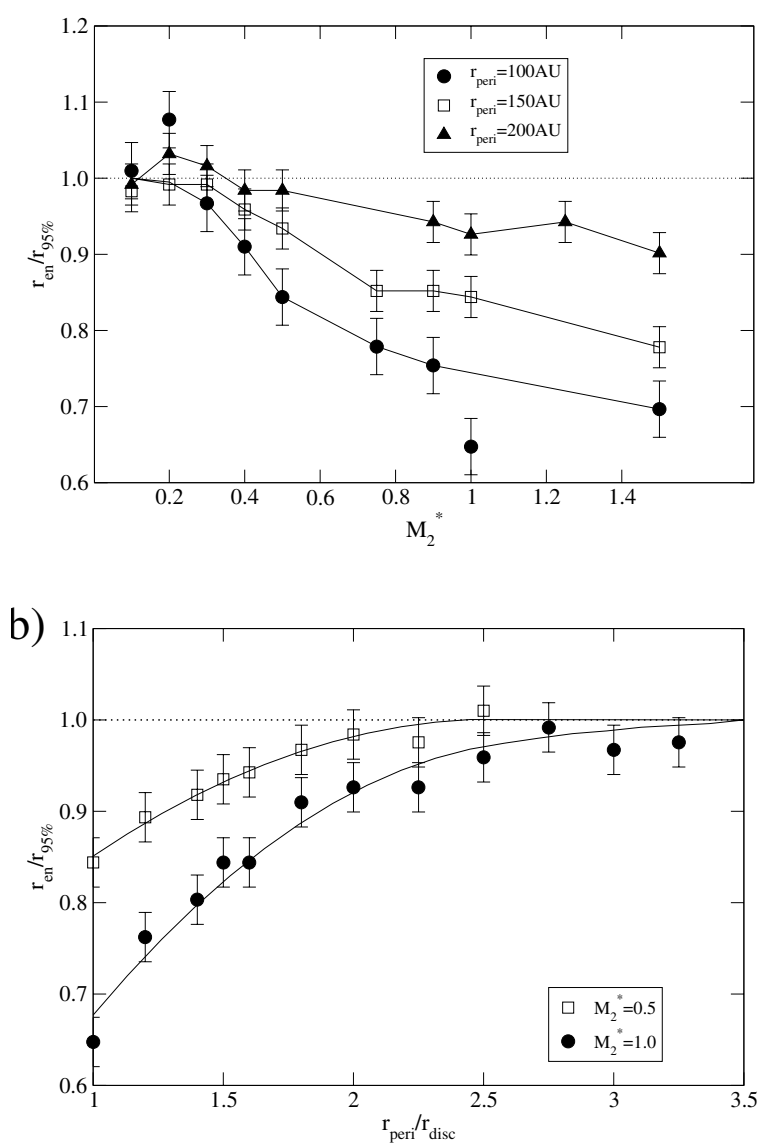

Fig. 9. Relative disc size after the encounter as a function of a) the mass of star 2 , b) the relative periastron $r_{\text {peri }} / r_{\text {initial. }}$. In a) the simulations were performed for $r_{\text {peri }}=100 \mathrm{AU}, 150 \mathrm{AU}$ and $200 \mathrm{AU}$. In b) the cases of $M_{2}^{*}=0.5 M_{\odot}$ and $M_{2}^{*}=1 M_{\odot}$ were investigated. $M_{2}^{*}$ is given in units of solar masses.

around $60 \%$ of the angular momentum are lost in an equal stellar mass encounters with $r_{\text {peri }} / r_{\text {disc }}=1$. Retrograde encounters lead to very little mass and angular momentum loss in the disc unless they are nearly grazing. Even for grazing encounters only high mass perturbers have a significant effect on the disc. For the prograde and retrograde case parabolic encounters are naturally most disruptive to the disc whereas hyperbolic encounters due to their shorter interaction time have less effect on the disc. Retrograde encounters seem to be more sensitive to deviations from the parabolic path, as even slightly non-parabolic encounters lead to significantly less mass and angular momentum loss.

In the case of non-coplanar encounters, up to an inclination of 45 degree between the path of star 2 and the disc plane of star 1 mass and angular momentum losses are slightly less than in the coplanar prograde case. However, for more inclined systems the mass and angular momentum loss drop significantly; for most encounters to zero, only for nearly grazing encounters there can be mass and angular momentum transport at such inclinations. For latter the minimum of mass and angular transport is not for the retrograde (180 degree) case, but somewhere between 120 and 165 degree. 
As far as possible fitting formulae for most of the above results are given. So that a much wider parameter space than previously is accessible.

In addition the capturing of mass by star 2 was investigated.

Our aim is to combine these results with simulations of dense clusters to be able to make predictions of the lifetime of disc in dense stellar clusters.

\section{References}

Balbus, S. A., \& Hawley, J. F. 2002, ApJ, 573

Bate, M. R., Bonnell, I. A., \& Bromm, V. 2003, MNRAS, 336

Boffin, H. M. J., Watkins, S. J., Bhattal, A. S., Francis, N., \& Whitworth, A. P. 1996, MNRAS, 300

Bonnell, I. A., Smith, K. W., Davies, M. B., \& Horne, K. 2001, MNRAS, 322

Hall, S. M. 1997, MNRAS, 287

Hall, S. M., Clarke, C. J., \& Pringle, J. E. 1996, MNRAS, 278

Heller, C. A. 1995, ApJ, 455
Jensen, E. L. N., Mathieu, R. D., Donar, A. X., \& Dullighan, A. 2004, ApJ, 600

Klahr, H. H., \& Bodenheimer, P. 2003, ApJ, 582

Kobayashi, H., \& Ida, S. 2001, 153

Nelson, R. P., Papaloizou, J. C. B., Larwood, J. D., \& Terquem, C. 1997, in Accretion Disks - New Aspects, Proc. of the EARA Workshop, ed. H. Meyer-Hofmeister, 182 (Heidelberg, Springer)

Olczak, C., Pfalzner, S., Eckart, A., in preparation

Ostriker, C. E. 1994, ApJ, 424, 292

Oxley, S., \& Woolfson, M. M. 2004, MNRAS, 348

Pfalzner, S. 2003, ApJ, 592

Pfalzner, S. 2004, ApJ, 602

Pfalzner, S., Umbreit, S., \& Henning, Th. 2005, ApJ, in press

Scally, A., \& Clarke, C. 2001, MNRAS, 325

Shu, F. H., Adams, F. C., \& Lizano, S. 1987, ARA\&A, 25

Stahler, S. W. 2000, in Proc. 33rd ESLAB Symp., Star formation from the Small to the Large scales, ESA SP-445, Noordwijk, 133

Stone, J. M., Gammie, C. F., Balbus, S. A., \& Hawley, J. F. 2000, in Protostars and Plantes IV, ed. V. Mannings, A. P. Boss, \& S. S. Russell, (Univ. of Arizona Press), 589 